\title{
Prediction of unstable anticoagulation with acenocoumarol versus warfarin in atrial fibrillation
}

\author{
Agata Hanna Bryk ${ }^{1,2}$, Krzysztof Plens ${ }^{3}$, Anetta Undas ${ }^{1,2}$ \\ ${ }^{1}$ Institute of Cardiology, Jagiellonian University Medical College, Kraków, Poland \\ ${ }^{2}$ John Paul II Hospital, Kraków, Poland \\ ${ }^{3}$ KCRI, Kraków, Poland
}

\begin{abstract}
Background: The $S A M e-T T_{2} R_{2}$ (sex female, age, medical history, treatment, tobacco use, race) score was developed in patients with atrial fibrillation $(A F)$ on warfarin. The present study aimed to 1) compare the anticoagulation quality and management of $A F$ patients treated with warfarin with those on acenocoumarol and 2) optimize the $S A M e-T T_{2} R_{2}$ score to detect $A F$ patients at high risk of unstable anticoagulation with acenocoumarol and warfarin.

Methods: In a single-center retrospective study, 320 patients with AF, including 15 (5\%) after valve replacement, aged 40-82 (median 70) years, including 203 (63\%) receiving acenocoumarol and 117 (37\%) treated with warfarin, were studied. The $S A M e-T T_{2} R_{2}$ score was modified based on the candidate factors retrieved from univariate regression and assessed using the receiver operating curves (ROC).

Results: A median SAMe-TT $T_{2} R_{2}$ score was 2 (1-3). Proportions of patients with $\geq 2$ points and $0-1$ points in the $S A M e-T T_{2} R_{2}$ score who had the time in therapeutic range $(T T R) \leq 70 \%$ were similar (61 [67\%] vs. 63 [56\%], $p=0.11$ ). A modified score, involving medical history (myocardial infarction [MI] and chronic obstructive pulmonary disease [COPD], 1 point), statin treatment (1 point) and tobacco use (2 points) had a higher area under the curve (AUC) in patients on acenocoumarol compared to SAMe$T_{2} R_{2}$ (0.66; 95\% confidence interval 0.58-0.73 vs. 0.56; 0.48-0.64, $\left.p=0.042\right) ; \geq 1$ point indicated TTR $>70 \%$ with a sensitivity and specificity of $61 \%$ and $63 \%$, respectively.

Conclusions: The $S A M e-T T_{2} R_{2}$ score is less effective in predicting unstable anticoagulation with acenocoumarol versus warfarin. Adding statin use and highlighting COPD and previous MI increases a predictive value of this score for acenocoumarol. (Cardiol J 2017; 24, 5: 477-483)
\end{abstract}

Key words: atrial fibrillation, anticoagulant therapy, acenocoumarol, warfarin

\section{Introduction}

The prevalence of atrial fibrillation (AF) has quadrupled over the last 50 years [1]. Although non-vitamin $\mathrm{K}$ antagonists have been introduced in therapy of $\mathrm{AF}$, vitamin $\mathrm{K}$ antagonists (VKA) remained the only treatment with established safety in valvular AF [2]. Quality of anticoagulation with VKA, assessed by the time in therapeutic range (TTR), is closely associated with the risk of stroke [3, 4]. Recently, several factors have been incorporated by Apostolakis et al. [5] into a clinical prediction scheme with the acronym SAMe-TT ${ }_{2} \mathrm{R}_{2}$ (sex female, age $>60$ years, medical history [more than two comorbidities], treatment [interacting drugs, e.g. amiodarone for rhythm control], tobacco use [doubled], race [doubled]).

The anticoagulants assessed by Apostolakis et al. [5] have been referred to as VKA; however, the population included in the AFFIRM (The Atrial Fibrillation Follow-up Investigation of Rhythm Management) study was treated only with war-

Address for correspondence: Anetta Undas, MD, PhD, Institute of Cardiology, Jagiellonian University Medical College, ul. Prądnicka 80, 31-202 Kraków, Poland, tel: +48 1261430 04, fax: +48 1261421 20, e-mail: mmundas@cyf-kr-edu.pl 
farin [6]. The SAMe-TT ${ }_{2} \mathrm{R}_{2}$ score has been demonstrated to have a modest ability of predicting stable anticoagulation control in patients treated with acenocoumarol [7], but there are no reports on the comparison of clinical utility of SAMe-TT ${ }_{2} R_{2}$ for warfarin and acenocoumarol. The differences observed in anticoagulation control dependent on the type of VKA may result from a shorter half-life of acenocoumarol compared to warfarin [8]. Apart from the pharmacokinetic profile, it has been shown that carriers of variant alleles for cytochrome $\mathrm{P}$ type 2C9 (CYP2C9) and gamma-glutamyl carboxylase genes required a lower dose of warfarin as compared with non-carriers; the effects of those polymorphisms have been absent with regard to acenocoumarol dose [9]. Arterial hypertension, use of statins and aspirin as well as older age were identified by Undas et al. [10] as the clinical factors responsible for improved anticoagulation control after switching from acenocoumarol to warfarin. Those observations might be especially interesting in regard to differences in utility of SAMe$-\mathrm{TT}_{2} \mathrm{R}_{2}$ for predicting stability of anticoagulation on warfarin and acenocoumarol, as the score is based on common clinical factors.

We aimed to: 1) compare the anticoagulation quality and management of AF patients treated with warfarin with those on acenocoumarol and 2) optimize the $S A M e-T_{2} R_{2}$ score to detect $A F$ patients at high risk of unstable anticoagulation with acenocoumarol and warfarin.

\section{Methods}

\section{Study design}

This single-center retrospective study included 320 consecutive patients with documented $\mathrm{AF}$, from the databases who were referred to the John Paul II Hospital, Krakow, Poland between 2010 and 2012. All patients were on oral anticoagulation with VKA for at least 6 months prior to enrolment. Paroxysmal AF, persistent AF and permanent $\mathrm{AF}$ were defined according to the $\mathrm{Eu}$ ropean Society of Cardiology guidelines [11]. The exclusion criteria were: signs of acute infection and C-reactive protein $\geq 10 \mathrm{mg} / \mathrm{L}$, acute coronary syndrome within the preceding 12 months being treated with dual antiplatelet therapy, hepatic injury (alanine aminotransferase $\geq 1.5$ times the upper limit of normal), chronic kidney disease stage 4 or more or known malignancy. The study was approved by the Ethical Committee of the Jagiellonian University. All participants gave informed consent.
Data on demographic characteristics, cardiovascular risk factors, comorbidities, prior valvular surgery and treatment were collected by the recruiting physician. Obesity was defined as body mass index $\geq 30 \mathrm{~kg} / \mathrm{m}^{2}$. Arterial hypertension was diagnosed based on a history of hypertension (consistent blood pressure $\geq 140 / 90 \mathrm{~mm} \mathrm{Hg}$ ). Diabetes mellitus was defined as according to the American Diabetic Association criteria [12]. Coronary artery disease was diagnosed based on positive results of cardiac stress tests, cardiac catheterisation, or coronary computed tomography angiography. Myocardial infarction (MI) was recognized according to the guidelines [13]. Heart failure was defined as the presence of relevant symptoms and signs and left ventricular ejection fraction $\leq 40 \%$. Ischemic stroke prior to the enrollment was diagnosed based on the symptoms and positive finding of brain noncontrast-enhanced computed tomography [14]. Chronic obstructive pulmonary disease (COPD) was diagnosed based on the signs and symptoms and results of spirometry. Chronic kidney disease was defined according to the guidelines [15]. TTR during VKA treatment was calculated with Rosendaal's method [16]. Stable anticoagulation was defined as having the TTR of $\geq 70 \%$ [17]. The value of $\geq 2$ points in SAMe-TT ${ }_{2} \mathrm{R}_{2}$ score (sex female, age $>60$ years, medical history [more than two comorbidities], treatment [interacting drugs, e.g. amiodarone for rhythm control], tobacco use [doubled], race [doubled]) indicated the risk of unstable anticoagulation [5]. The $\mathrm{CHA}_{2} \mathrm{DS}_{2}$-VASc (cardiac failure, hypertension, age $>75$ years, diabetes, stroke, vascular disease, age 65-74 and sex) score was used to assess the risk of stroke.

\section{Statistical analysis}

Categorical variables were presented as numbers and percentages. Continuous variables were expressed as mean \pm standard deviation (SD) or median and interquartile range. Normality was assessed by the Shapiro-Wilk test. Equality of variance was assessed using the Levene's test. Differences between groups were compared using the Student's or the Welch's t-test depending on equality of variance for normally distributed variables. The Mann-Whitney U test was used for non-normally distributed continuous variables. Categorical variables were compared by the Fisher's exact test for $2 \times 2$ contingency tables and Pearson's $\chi^{2}$ test for other tables.

To assess the predictive ability of identifying the unstable patients with the SAMe-TT $\mathrm{R}_{2}$ score, compared herein was the percentage of patients with 
TTR $\leq 70 \%$ among patients with $\mathrm{SAMe}^{-\mathrm{TT}_{2}} \mathrm{R}_{2} \geq 2$ vs. $0-1$. The univariate logistic regression was performed to calculate the odds ratio (OR) and $95 \%$ confidence interval $(95 \% \mathrm{CI})$ of achieving TTR $>70 \%$ for the following factors. Covariates associated with TTR at levels of 0.1 in univariate analysis served as candidate factors to build the modified model, where each factor was given 1 or 2 points. The modified models were further tested in binary logistic regression models using a TTR cut-off at $70 \%$. The predictive ability of the modified score was compared with SAMe-TT $\mathrm{R}_{2}$ score using area under the receiver operating curve (AUC). The sensitivity and specificity were calculated. P-values $<0.05$ were considered statistically significant. The study was powered to have a $80 \%$ chance of detecting a 2-4\% TTR difference between groups stratified by SAMe-TT ${ }_{2} \mathrm{R}_{2}$ score of $0-1,2-3$ and $\geq 4$ using a p-value of 0.05 . In order to demonstrate such a difference or greater, 102 patients were required in each group. All calculations were done with JMP ${ }^{\circledR}$, Version 9.0.0 SAS Institute Inc., Cary, NC, 1989-2007.

\section{Results}

The final analysis included 320 patients, 203 $(63 \%)$ receiving acenocoumarol and 117 (37\%) treated with warfarin. There were 305 patients with non-valvular AF, 11 (3\%) subjects after mitral valve replacement, 3 (1\%) following aortic valve replacement and 1 patient who underwent concomitant mitral and aortic valve replacement. The median age was 70 (minimum 46, maximum 89). The majority of the patients studied represented overweight women with permanent $\mathrm{AF}$ at high risk of ischemic stroke in $\mathrm{CHA}_{2} \mathrm{DS}_{2}$-VASc (Table 1). Patients treated with acenocoumarol were slightly younger, less frequently smoked cigarettes and were diagnosed with COPD compared to these receiving warfarin (Table 1 ).

\section{SAMe-TT ${ }_{2} \mathbf{R}_{2}$}

A median SAMe-TT ${ }_{2} \mathrm{R}_{2}$ score was 2 (1-3). Ninety one (45\%) patients on acenocoumarol and $77(66 \%)$ on warfarin had SAMe-TT $\mathrm{R}_{2} \geq 2(\mathrm{p}=$ $=0.0003)$. The median TTR was 66 (58-75.5)\% with no differences related to the type of VKA. Median TTR of patients with SAMe-TT ${ }_{2} \mathrm{R}_{2} \geq 2$ ( $\mathrm{n}=186$, $52 \%$ ) was lower by $9.6 \%$ compared to those with

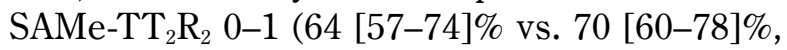
$\mathrm{p}=0.002)$. There was a progressive decline in median TTR from the score of $0-1$ to $\geq 4$ in SAMe-TT ${ }_{2} \mathrm{R}_{2}$ score in the whole cohort of patients
(70 [60-78]\% vs. $65[57-75] \%$ vs. $61[54.5-70] \%$, $\mathrm{p}=0.007)$. The same was true for the patients treated with acenocoumarol (69 [59-77.5]\% vs. 65 $[57-75] \%$ vs. $55.5[45.75-61.75] \%, \mathrm{p}=0.009)$ and warfarin $(71.5[63.25-79] \%$ vs. $63[56.5-73] \%$ vs. 63.5 [58.25-72.25]\%, $\mathrm{p}=0.05$ ) (Fig. 1).

124 patients $(39 \%)$ had TTR $>70 \%$. Among $168(52 \%)$ patients with SAMe-TT ${ }_{2} \mathrm{R}_{2} \geq 2$ there were $115(68 \%)$ individuals with TTR $\leq 70 \%$. Among patients treated with acenocoumarol, there was a similar proportion of patients with TTR $\leq 70 \%$ among those SAMe-TT $\mathrm{R}_{2} \geq 2$ points as well as $0-1$ points $(61[67] \%$ vs. 63 [56]\%, $\mathrm{p}=0.1)$. In contrast, taking into consideration only warfarintreated patients, there were more subjects with

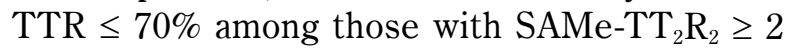
points in comparison to $0-1$ points $(54$ [70]\% vs. $18[45] \%, \mathrm{p}=0.01)$.

\section{Modified SAMe-TT ${ }_{2} \mathbf{R}_{2}$}

The following candidate factors found in the univariate regression analysis for TTR $>70 \%$ (Table 2) were included in the modified score for acenocoumarol-treated patients: medical history ( $\mathrm{MI}-1$ point; COPD -1 point), treatment (statins -1 point), tobacco use ( 2 points). In acenocoumarol-treated patients, the modified score predicting the TTR $>70 \%$ had increased AUC compared to the $\mathrm{SAMeTT}_{2} \mathrm{R}_{2}(0.66 ; 95 \% \mathrm{CI}$ $0.58-0.73$ vs. 0.56 ; $95 \%$ CI $0.48-0.64, \mathrm{p}=0.04)$. The score of $\geq 1$ point was associated with a TTR $>70 \%$ with a sensitivity and specificity of $61 \%$ and $63 \%$, respectively. In the warfarin-treated patients, the AUC of identifying the TTR $>70 \%$ by the modified model was comparable with SAMe$-\mathrm{TT}_{2} \mathrm{R}_{2}$ (AUC $0.61 ; 95 \%$ CI $0.51-0.71$ vs. $0.65 ; 95 \%$ CI $0.55-0.75, \mathrm{p}=0.3)$.

\section{Discussion}

According to the present research of available literature, this study is the first to compare the predictive ability of SAMe-TT $\mathrm{R}_{2}$ score in AF patients treated with acenocoumarol versus warfarin on a long-term basis. This study showed that common and clinical risk factors incorporated in the SAMe-TT ${ }_{2} \mathrm{R}_{2}$ score are less effective in predicting unstable anticoagulation in AF patients on acenocoumarol as compared to warfarin. The modified score highlights the impact of statins, previous MI and COPD on the quality of acenocoumarol treatment in patients with $\mathrm{AF}$.

Previously, Roldan et al. [18] validated the clinical value of the SAMe-TT $\mathrm{R}_{2}$ score in a prospec- 
Table 1. Baseline characteristics of patients with atrial fibrillation (AF) treated with acenocoumarol and warfarin.

\begin{tabular}{|c|c|c|c|}
\hline Variable & $\begin{array}{l}\text { Acenocoumarol } \\
(n=203,63 \%)\end{array}$ & $\begin{array}{c}\text { Warfarin } \\
(\mathrm{n}=117,37 \%)\end{array}$ & $\mathbf{P}$ \\
\hline \multicolumn{4}{|l|}{ Demographic characteristics: } \\
\hline Male & $89(44 \%)$ & $53(45 \%)$ & 0.8 \\
\hline Age [years] & $69(62-73)$ & $72(65-78)$ & 0.006 \\
\hline BMI $\left[\mathrm{kg} / \mathrm{m}^{2}\right]$ & $28(24.6-32.6)$ & $27.25(24.5-31)$ & 0.4 \\
\hline Tobacco use & $23(11)$ & $58(50)$ & $<0.001$ \\
\hline \multicolumn{4}{|l|}{ Atrial fibrillation - type: } \\
\hline Permanent & $91(44 \%)$ & $54(46 \%)$ & 0.2 \\
\hline Persistent & $55(27 \%)$ & $40(34 \%)$ & \\
\hline Paroxysmal & $57(28 \%)$ & $23(20 \%)$ & \\
\hline Time since AF diagnosis [years] & $5(3-9)$ & $4.0(3-10)$ & 0.8 \\
\hline \multicolumn{4}{|l|}{ Comorbidities: } \\
\hline Coronary artery disease & $60(30 \%)$ & $20(17 \%)$ & 0.02 \\
\hline Myocardial infarction & $35(17 \%)$ & $19(16 \%)$ & 0.9 \\
\hline Peripheral artery disease & $8(4 \%)$ & $28(24 \%)$ & $<0.001$ \\
\hline Arterial hypertension & $118(58 \%)$ & $62(53 \%)$ & 0.4 \\
\hline Heart failure & $50(25 \%)$ & $19(16 \%)$ & 0.09 \\
\hline Stroke & $25(12 \%)$ & $20(17 \%)$ & 0.2 \\
\hline COPD & $21(10 \%)$ & $34(29 \%)$ & $<0.001$ \\
\hline Diabetes mellitus type 2 & $34(17 \%)$ & $21(18 \%)$ & 0.9 \\
\hline Chronic kidney disease & $25(12 \%)$ & $22(19 \%)$ & 0.1 \\
\hline \multicolumn{4}{|l|}{ Medication: } \\
\hline VKA weekly dose [mg/week] & $32.0(23.0-42.0)$ & $35.0(21.0-48.0)$ & - \\
\hline TTR [\%] & $66.5 \pm 14.3$ & $66.7 \pm 13.3$ & 0.09 \\
\hline Acetylsalicylic acid & $60(30 \%)$ & $20(17 \%)$ & 0.02 \\
\hline Clopidogrel & $10(5 \%)$ & $3(3 \%)$ & 0.4 \\
\hline Beta-blocker & $113(56 \%)$ & $57(49 \%)$ & 0.2 \\
\hline ACEI & $76(37 \%)$ & $40(34 \%)$ & 0.6 \\
\hline Statin & $83(41 \%)$ & $44(38 \%)$ & 0.6 \\
\hline Amiodarone & $26(13 \%)$ & $9(8 \%)$ & 0.2 \\
\hline Digoxin & $29(14 \%)$ & $15(13 \%)$ & 0.9 \\
\hline \multicolumn{4}{|l|}{ Scores: } \\
\hline $\mathrm{SAMe}-\mathrm{TT}_{2} \mathrm{R}_{2}$ & $1(1-2)$ & $2(1-3)$ & $<0.001$ \\
\hline SAMe-TT ${ }_{2} R_{2} \geq 2$ & $38(19 \%)$ & $49(42 \%)$ & $<0.001$ \\
\hline $\mathrm{CHA}_{2} \mathrm{DS}_{2}$-VASc & $3(2-4)$ & $3(2-4)$ & 0.5 \\
\hline $\mathrm{CHA}_{2} \mathrm{DS}_{2}-\mathrm{VASc} \geq 2$ & $164(81 \%)$ & $97(83 \%)$ & 0.1 \\
\hline $\mathrm{CHA}_{2} \mathrm{DS}_{2}-\mathrm{VASc}=1$ & $29(14 \%)$ & $17(14 \%)$ & 0.6 \\
\hline $\mathrm{CHA}_{2} \mathrm{DS}_{2}-\mathrm{VASc}=0$ & $10(5 \%)$ & $3(3 \%)$ & 0.5 \\
\hline
\end{tabular}

Data are given as number (percentage) or mean \pm standard deviation or median (interquartile range). Results are presented as the p-value for Fisher's exact test for categorical variables or t-Student's test for normally distributed variables or Mann-Whitney's test for other variables. $\mathrm{BMI}$ - body mass index; COPD — chronic obstructive pulmonary disease; VKA - vitamin K antagonists; TTR - time in therapeutic range; ACEI - angiotensin converting enzyme inhibitor; SAMe- $\mathrm{TT}_{2} \mathrm{R}_{2}$ - sex female, age, medical history, treatment, tobacco use, race score; $\mathrm{CHA}_{2} \mathrm{DS}_{2}$-VASc - cardiac failure, hypertension, age $>75$ years, diabetes, stroke, vascular disease, age 65-74 and sex score

tive "real-world" cohort of 459 patients initiating oral anticoagulation with acenocoumarol. In a latter study patients with less than 2 points in SAMe-
$-\mathrm{TT}_{2} \mathrm{R}_{2}$ score had TTR lower by $6 \%$ than those with more than 2 points, indicated that the score properly identified the individuals on acenocoumarol [18]. 


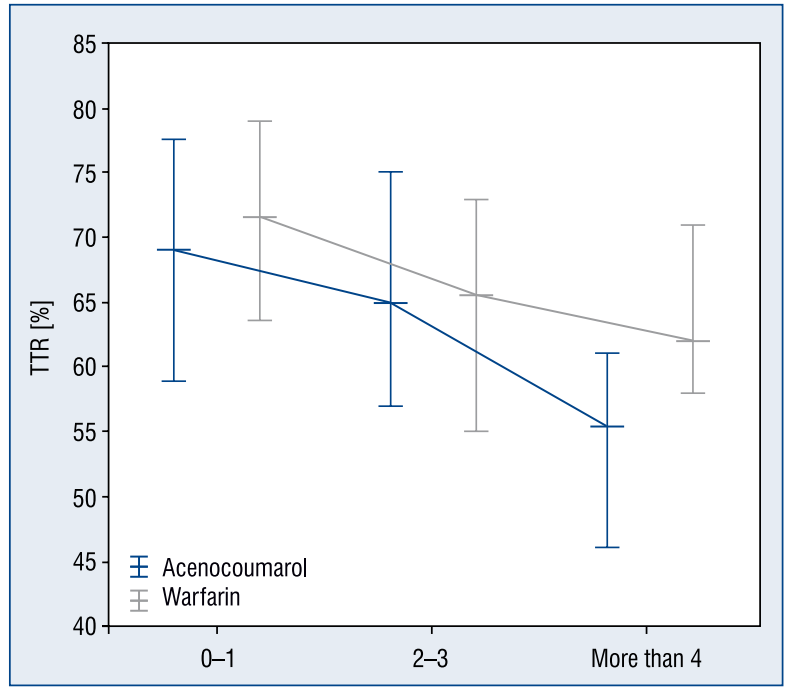

Figure 1. Comparison of the median time in therapeutic range (TTR) between patients treated acenocoumarol and warfarin with $0-1,2-3$ and more than 4 points in SAMe- $\mathrm{TT}_{2} \mathrm{R}_{2}$ (sex, age $<60$ years, more than two comorbidities, interacting drugs, tobacco and race) score. Vertical lines extend from the $25^{\text {th }}$ to the $75^{\text {th }}$ percentiles, with the horizontal lines in the middle at the median value.
However, in a subsequent Spanish study involving 1524 patients, among them approximately $95 \%$ on acenocoumarol, the discriminating capability of the SAMe-TT ${ }_{2} \mathrm{R}_{2}$ score was modest (AUC 0.59) and did not improve after adding new factors [7].

In this study the percentage of AF patients with TTR less than $70 \%$ was comparable among individuals with $\mathrm{SAMe}_{\mathrm{TT}} \mathrm{R}_{2}$ over 2 points and $0-1$ points. It suggested that $S A M e-T_{2} R_{2}$ score was not able to identify the AF patients treated with acenocoumarol at risk of poor anticoagulation control using the TTR cut-off value of $70 \%$. It seems that the clinical factors predispose to unstable anticoagulation to a different extent during treatment with warfarin and acenocoumarol. It was found that COPD, reported to coexist in a considerable percentage of AF patients (10\%) [19], strongly contributed to the prediction of the anticoagulation stability. A potential factor which might explain the association between COPD and stability of anticoagulation with acenocoumarol is drug-drug interaction. Patients with COPD exacerbation commonly receive corticosteroids and macrolides or fluoroquinolones [20], both interacting with the anticoagulant

Table 2. Factors associated with time in therapeutic range (TTR) $>70 \%$ in patients on acenocoumarol.

\begin{tabular}{|c|c|c|}
\hline Variable & OR $(95 \% \mathrm{Cl})$ & $\mathbf{P}$ \\
\hline \multicolumn{3}{|l|}{ Demographic data: } \\
\hline Male gender & $0.80(0.45-1.41)$ & 0.4 \\
\hline Age $<60$ years & $1.25(0.60-2.56)$ & 0.5 \\
\hline Tobacco use & $0.13(0.02-0.45)$ & $<0.001$ \\
\hline \multicolumn{3}{|l|}{ Comorbidities: } \\
\hline Arterial hypertension & $0.92(0.52-1.64)$ & 0.8 \\
\hline Myocardial infarction & $0.48(0.20-1.06)$ & 0.07 \\
\hline Peripheral artery disease & $0.51(0.07-2.28)$ & 0.4 \\
\hline Heart failure & $0.95(0.49-1.82)$ & 0.9 \\
\hline Stroke & $1.53(0.65-3.56)$ & 0.3 \\
\hline COPD & $0.07(0.00-0.33)$ & $<0.001$ \\
\hline Diabetes mellitus type 2 & $1.12(0.52-2.36)$ & 0.8 \\
\hline Chronic kidney disease & $0.57(0.21-1.39)$ & 0.2 \\
\hline \multicolumn{3}{|l|}{ Medication: } \\
\hline Acetylsalicylic acid & $0.97(0.52-1.79)$ & 0.9 \\
\hline Clopidogrel & $0.66(0.14-2.45)$ & 0.5 \\
\hline Beta-blockers & $0.85(0.48-1.50)$ & 0.6 \\
\hline ACEI & $0.79(0.44-1.43)$ & 0.4 \\
\hline Statin & $0.53(0.29-0.94)$ & 0.031 \\
\hline Digoxin & $1.13(0.50-2.49)$ & 0.8 \\
\hline Amiodarone & $0.81(0.33-1.88)$ & 0.6 \\
\hline
\end{tabular}

Data presented as the odds ratio (OR) and 95\% confidence interval (Cl) and p-value calculated using the logistic univariate regression analysis; COPD - chronic obstructive pulmonary disease; ACEI - angiotensin-converting enzyme inhibitor 
treatment [21]. Here might be the point where the shorter half-live of acenocoumarol may not entirely justify but may exaggerate the effect of interacting drugs on the acenocoumarol pharmacokinetics in comparison to warfarin.

The present study emphasised the role of the previous $\mathrm{MI}$ in maintaining stable anticoagulation. $\mathrm{MI}$ is an established risk factor for $\mathrm{AF}$, with $\mathrm{AF}$ occurring in $6 \%$ to $21 \%$ of patients following MI [22, 23]. The poor TTR control was an independent predictor of major adverse cardiovascular events including $\mathrm{MI}$ in a cohort of $627 \mathrm{AF}$ patients on VKA patients followed by Pastori et al. [24]. We assume that a decreased TTR prior to the MI may reflect the overall worse anticoagulation control observed in that subset of patients not only prior to but also following the MI. Mechanisms linking a history of MI with anticoagulation control need to be further investigated.

Unexpectedly, in the present study, statin use has been identified to be a risk factor for unstable anticoagulation with acenocoumarol. Previously, statins have been associated with the $9 \%$ lower risk of bleeding during VKA therapy [25], which suggested beneficial influence of statins on the anticoagulation stability. Statins favourably modulate blood coagulation by exerting mild anticoagulant and antiplatelet effects [26, 27]. The results of statins on blood clotting can also be observed in patients with $\mathrm{AF}$ [28]. In the context of VKA, drug-drug interactions may be however of key importance. Simvastatin has been found to reduce warfarin dose requirement by $29 \%$ in the CYP2C9*3 allele carriers compared with 5\% in non-carriers [29]. This effect may predispose unstable anticoagulation in patients carrying the CYP2C9*3 allele that represent $12 \%$ of Poles [9]. Further investigations are required to establish mechanisms behind the relationship between statin use and stability of anticoagulation with warfarin and acenocoumarol. Other postulated beneficial influences of statin is slowing the evolution of $\mathrm{AF}$ and reducing inflammation and fibrosis [30].

\section{Limitations of the study}

Several study limitations should be acknowledged. Firstly, baseline characteristics of the patients treated with acenocoumarol and warfarin in this study differed which could affect to some extent the differences observed in the discriminating ability of the scores. Secondly, the number of the studied patients was relatively low, although the study was sufficiently powered to show some intergroup differences. These findings cannot be reliably extrapolated to patients excluded from our analysis including those with advanced renal insufficiency or on dual antiplatelet therapy, as well as for predicting quality of anticoagulation with lower TTR out-offs [31]. Long-term follow-up to assess clinical impact of the scoring systems in terms of stroke and risk of bleeding was beyond the scope of the present study.

\section{Conclusions}

1. The SAMe-TT ${ }_{2} \mathrm{R}_{2}$ score appears less effective in predicting unstable anticoagulation with acenocoumarol compared with warfarin.

2. Adding the statin use and highlighting a role of COPD and previous MI in the modified score increase its predictive value for acenocoumarol.

Conflict of interest: None declared

\section{References}

1. Schnabel RB, Yin X, Gona P, et al. 50 year trends in atrial fibrillation prevalence, incidence, risk factors, and mortality in the Framingham Heart Study: a cohort study. Lancet. 2015; 386(9989): 154-162, doi: 10.1016/S0140-6736(14)61774-8, indexed in Pubmed: 25960110.

2. Kirchhof P, Benussi S, Kotecha D, et al. 2016 ESC Guidelines for the management of atrial fibrillation developed in collaboration with EACTS. Eur Heart J. 2016; 37(38): 2893-2962, doi: 10.1093/eurheartj/ehw210, indexed in Pubmed: 27567408.

3. Gallagher AM, Setakis E, Plumb JM, et al. Risks of stroke and mortality associated with suboptimal anticoagulation in atrial fibrillation patients. Thromb Haemost. 2011; 106(5): 968-977, doi: 10.1160/TH11-05-0353, indexed in Pubmed: 21901239.

4. Kosior DA. Risk stratification schemes for stroke in atrial fibrillation: the predictive factors still undefined. Pol Arch Med Wewn. 2015; 125(12): 889-890, indexed in Pubmed: 26787631.

5. Apostolakis S, Sullivan RM, Olshansky B, et al. Factors affecting quality of anticoagulation control among patients with atrial fibrillation on warfarin: the $\mathrm{SAMe}_{\mathrm{TT}} \mathrm{R}_{2}$ score. Chest. 2013; 144(5): 1555-1563, doi: 10.1378/chest.13-0054, indexed in Pubmed: 23669885.

6. Atrial fibrillation follow-up investigation of rhythm management - the AFFIRM study design. The Planning and Steering Committees of the AFFIRM study for the NHLBI AFFIRM investigators. Am J Cardiol. 1997; 79(9): 1198-1202, indexed in Pubmed: 9164885.

7. Lobos-Bejarano JM, Barrios V, Polo-García J, et al. on behalf the PAULA Study Team. Evaluation of SAMe-TT2R2 score and other clinical factors influencing the quality of anticoagulation therapy in non-valvular atrial fibrillation: a nationwide study in Spain. Curr Med Res Opin. 2016; 32(7): 1201-1207, doi: 10.1185/03007 995.2016.1164676, indexed in Pubmed: 26967541.

8. Pattacini C, Manotti C, Pini M, et al. A comparative study on the quality of oral anticoagulant therapy (warfarin versus aceno- 
coumarol). Thromb Haemost. 1994; 71(2): 188-191, indexed in Pubmed: 8191397.

9. Wypasek E, Branicka A, Awsiuk M, et al. Genetic determinants of acenocoumarol and warfarin maintenance dose requirements in Slavic population: a potential role of CYP4F2 and GGCX polymorphisms. Thromb Res. 2014; 134(3): 604-609, doi: 10.1016/j. thromres.2014.06.022, indexed in Pubmed: 25042728.

10. Undas A, Cieśla-Dul M, Zółciński M, et al. Switching from acenocoumarol to warfarin in patients with unstable anticoagulation and its effect on anticoagulation control. Pol Arch Med Wewn. 2009; 119(6): 360-365, indexed in Pubmed: 19694217.

11. Camm AJ, Kirchhof P, Lip GYH, et al. Guidelines for the management of atrial fibrillation The Task Force for the Management of Atrial Fibrillation of the Developed with the special contribution of the European Heart Rhythm Association. Eur Heart J. 2010; 31(19): 2369-2429, doi: 10.1093/eurheartj/ehq278.

12. 2. Classification and Diagnosis of Diabetes. Diabetes Care. 2015; 38(Supplement 1): S8-S16, doi: 10.2337/dc15-S005.

13. Thygesen K, Alpert JS, White HD. W imieniu Wspólnej Komisji Specjalnej ESC/ACCF/AHA/WHF ds. nowelizacji definicji zawału serca. Uniwersalna definicja zawalu serca. Kardiol Pol. 2008; 66: 47-62.

14. Jauch EC, Saver JL, Adams HP, et al. American Heart Association Stroke Council, Council on Cardiovascular Nursing, Council on Peripheral Vascular Disease, Council on Clinical Cardiology. Guidelines for the early management of patients with acute ischemic stroke: a guideline for healthcare professionals from the American Heart Association/American Stroke Association. Stroke. 2013; 44(3): 870-947, doi: 10.1161/ /STR.0b013e318284056a, indexed in Pubmed: 23370205.

15. National Kidney Foundation. K/DOQI clinical practice guidelines for chronic kidney disease: evaluation, classification, and stratification. Am J Kidney Dis. 2002; 39(2 Suppl 1): S1-S266, indexed in Pubmed: 11904577.

16. Rosendaal FR, Cannegieter SC, van der Meer FJ, et al. A method to determine the optimal intensity of oral anticoagulant therapy. Thromb Haemost. 1993; 69(3): 236-239, indexed in Pubmed: 8470047.

17. Dallalzadeh LO, Go AS, Chang Y, et al. Stability of high-quality warfarin anticoagulation in a community-based atrial fibrillation cohort: The Anticoagulation and Risk Factors in Atrial Fibrillation (ATRIA) Study. J Am Heart Assoc. 2016; 5(7), doi: 10.1161/ /JAHA.116.003482, indexed in Pubmed: 27451456.

18. Roldán V, Cancio S, Gálvez J, et al. The SAMe-TT2R2 score predicts poor anticoagulation control in AF patients: A prospective ,Real-world' Inception Cohort Study. Am J Med. 2015; 128(11): 1237-1243, doi: 10.1016/j.amjmed.2015.05.036, indexed in Pubmed: 26087049.

19. Singer DE, Hellkamp AS, Piccini JP, et al. ROCKET AF Investigators. Impact of global geographic region on time in therapeutic range on warfarin anticoagulant therapy: data from the ROCKET AF clinical trial. J Am Heart Assoc. 2013; 2(1): e000067, doi: 10.1161/JAHA.112.000067, indexed in Pubmed: 23525418.
20. Qureshi H, Sharafkhaneh A, Hanania NA. Chronic obstructive pulmonary disease exacerbations: latest evidence and clinical implications. Ther Adv Chronic Dis. 2014; 5(5): 212-227, doi: 10.1177/2040622314532862, indexed in Pubmed: 25177479.

21. Hazlewood KA, Fugate SE, Harrison DL. Effect of oral corticosteroids on chronic warfarin therapy. Ann Pharmacother. 2006; 40(12): 2101-2106, doi: 10.1345/aph.1H418, indexed in Pubmed: 17119104.

22. Soliman EZ, Safford MM, Muntner P, et al. Atrial fibrillation and the risk of myocardial infarction. JAMA Intern Med. 2014; 174(1): 107-114, doi: 10.1001/jamainternmed.2013.11912, indexed in Pubmed: 24190540.

23. Zielonka A, Tkaczyszyn M, Mende M, et al. Atrial fibrillation in outpatients with stable coronary artery disease: results from the multicenter RECENT study. Pol Arch Med Wewn. 2015; 125(3): 162-171, indexed in Pubmed: 25644126.

24. Pastori D, Pignatelli P, Saliola M, et al. Inadequate anticoagulation by vitamin $\mathrm{K}$ antagonists is associated with major adverse cardiovascular events in patients with atrial fibrillation. Int J Cardiol. 2015; 201: 513-516, doi: 10.1016/j.ijcard.2015.08.054, indexed in Pubmed: 26318513.

25. van Rein N, Cannegieter SC, le Cessie S, et al. Statins and risk of bleeding: an analysis to evaluate possible bias due to prevalent users and healthy user aspects. Am J Epidemiol. 2016; 183(10): 930-936, doi: 10.1093/aje/kwv255, indexed in Pubmed: 27189329 .

26. Undas A, Brummel-Ziedins KE, Mann KG. Anticoagulant effects of statins and their clinical implications. Thromb Haemost. 2014; 111(3): 392-400, doi: 10.1160/TH13-08-0720, indexed in Pubmed: 24285296.

27. Undas A, Brummel-Ziedins KE, Mann KG. Statins and blood coagulation. Arterioscler Thromb Vasc Biol. 2005; 25(2): 287294, doi: 10.1161/01.ATV.0000151647.14923.ec, indexed in Pubmed: 15569822

28. van Kuilenburg J, Lappegård KT, Sexton J, et al. Persisting thrombin activity in elderly patients with atrial fibrillation on oral anticoagulation is decreased by anti-inflammatory therapy with intensive cholesterol-lowering treatment. J Clin Lipidol. 2011; 5(4): 273-280, doi: 10.1016/j.jacl.2011.05.003, indexed in Pubmed: 21784372.

29. Andersson ML, Eliasson E, Lindh JD. A clinically significant interaction between warfarin and simvastatin is unique to carriers of the CYP2C9*3 allele. Pharmacogenomics. 2012; 13(7): 757-762, doi: 10.2217/pgs.12.40, indexed in Pubmed: 22594507.

30. Cocco G, Jerie P. New concepts in the therapy of atrial fibrillation. Cardiol J. 2016; 23(1): 3-11, doi: 10.5603/CJ.a2015.0053, indexed in Pubmed: 26412599.

31. Turk UO, Tuncer E, Alioglu E, et al. Evaluation of the impact of warfarin time in therapeutic range on outcomes of patients with atrial fibrillation in Turkey: perspectives from the observational, prospective WATER Registry. Cardiol J. 2015; 22(5): 567-575, doi: 10.5603/CJ.a2015.0035, indexed in Pubmed: 26100825. 